\title{
The Performance of Redescending M-Estimators when Outliers are in Two Dimensional Space
}

\author{
Anekwe Stella Ebele ${ }^{1, *}$ and Onyeagu Sidney Iheanyi ${ }^{2}$ \\ ${ }^{1}$ Department of Statistics, Nnamdi Azikiwe University, Awka, Nigeria \\ e-mail: stellaanekwe@gmail.com \\ ${ }^{2}$ Department of Statistics, Nnamdi Azikiwe University, Awka, Nigeria \\ e-mail: si.onyeagu@unizik.edu.ng
}

\begin{abstract}
M-estimators are robust estimators that give less weight to the observations that are outliers while redescending M-estimators are those estimators that are built such that extreme outliers are completely rejected. In this paper, redescending M-estimators are compared using both the Monte Carlo simulation method and the real life data to ascertain the method that is more efficient and robust when outliers are in both $x$ and $y$ directions. The results from the simulation study and the real life data indicate that Anekwe redescending M-estimator is more efficient and robust when outliers are in both $x$ and $y$ directions.
\end{abstract}

\section{Introduction}

\subsection{Redescending M-estimators}

Redescending M-estimators are estimators with their influence functions $(\psi$ functions) redescending to zero. They are those M-estimators that reject extreme outliers. Some of these estimators are:

\subsubsection{Hampel redescending M-estimator}

Hampel's three-part redescending M-estimator was proposed by [7] in the Princeton Robustness study. Its $\psi$-function is given as

Received: November 18, 2021; Revised: December 9, 2021; Accepted: December 14, 2021

2020 Mathematics Subject Classification: 49-XX, 49J15, 49K15, 49Mxx.

Keywords and phrases: outliers, robustness, M-estimators, redescending M-estimators, efficiency. 


$$
\psi(r)=\left\{\begin{array}{cl}
r & ; \text { if }|r| \leq a \\
a \operatorname{sign}(r) & ; \text { if } a<|r| \leq b \\
\frac{(c-|r|)}{(c-b)} a \operatorname{sign}(r) & ; \text { if } b<|r| \leq c \\
0 & ; \text { if }|r|>c
\end{array}\right.
$$

where $a, b, c$ are positive constants and $0<a \leq b<c<\infty$ and $r$ are the residuals scaledover Median Absolute Deviation MAD.

\subsubsection{Tukey's biweight redescending M-estimator}

[4] proposed Tukey's biweight M-estimator and its $\psi$-function is given as

$$
\psi(r)=\left\{\begin{array}{cl}
r\left\{1-\left(\frac{r}{c}\right)^{2}\right\}^{2} & ;|r| \leq c \\
0 & ; \text { otherwise }
\end{array}\right.
$$

where $c$ is arbitrary value known as tuning constant and $r$ are the residuals scaled over MAD. For Tukey's biweight, $c=4.685$ gives $95 \%$ efficiency on normal distribution.

\subsubsection{Alarm redescending M-estimator}

[1] proposed the Alarm's Redescending M-estimator for robust regression and outlier detection. Its $\psi$-function is given as

$$
\psi(r)=\left\{\begin{array}{cl}
\frac{16 r\left(e^{-(r / c)^{2}}\right.}{\left(1+e^{-(r / c)^{4}}\right.} & ;|r| \leq c \\
0 & ;|r|>c
\end{array}\right.
$$

where $c$ is the tuning constant and $r$ are the residuals scaled over MAD.

\subsubsection{Anekwe redescending M-estimator}

[3] proposed the Anekwe's redescending M-estimator for robust regression and outlier detection. Its $\psi$-function is given as

$$
\psi(r)=\left\{\begin{array}{cl}
r\left(1-\left(\frac{r}{c}\right)^{2}\right)^{2}\left(1+\left(\frac{r}{c}\right)^{2}\right)^{2} & ;|r|<c \\
0 & ;|r| \geq c
\end{array}\right.
$$

where $c$ is the tuning constant for the $i t h$ observation and the variable $r$ are the residuals scaled over MAD.

\section{Simulation Design}

Monte Carlo simulation method is used to generate random data from different 
probability distributions. We took the true parameters to be 1,2 , and 5 for $\beta_{0}, \beta_{1}$, and $\beta_{2}$ respectively. Each simulation case was replicated $M=1000$ times. The estimates of each estimator were calculated in each of the iteration and the Mean of the M replicated estimates given by

$$
\hat{\beta}_{j}=\frac{\sum_{i=1}^{M} \hat{\beta}_{j i}}{M} \quad \text { for } j=0,1,2, \cdots, p
$$

was recorded for each estimator.

For comparison, the parameters estimates of the Mean Square Error (MSE) and the absolute bias (BIAS) of the [7], [4] and [1] and the [3] redescending M-estimators are computed.

Robustness of an estimator is measured using absolute bias given as

$$
\operatorname{Abs\operatorname {Bias}}\left(\hat{\beta}_{j}\right)=\left|\beta_{j}-\hat{\beta}_{j}\right| \quad \text { for } j=0,1,2, \cdots, p
$$

Efficiency of an estimator is measured using the MSE (mean square error) defined as

$$
\operatorname{MSE}\left(\hat{\beta}_{j}\right)=\frac{\sum_{i=1}^{M}\left(\beta_{j}-\hat{\beta}_{j i}\right)^{2}}{M} \quad \text { for } j=0,1,2, \cdots, p
$$

and the variance of the estimator is defined as

$$
\operatorname{Var}\left(\hat{\beta}_{j}\right)=\operatorname{MSE}\left(\hat{\beta}_{j}\right)-\left(\operatorname{Bias}\left(\hat{\beta}_{j}\right)\right)^{2} \quad \text { for } j=0,1,2, \cdots, p .
$$

The estimator with lowest MSE is the most efficient; the smaller the MSE the more efficient is the estimator.

Simulated data were generated (including percentage mixtures of contaminated and uncontaminated data) in simple and multiple regressions, using two sample sizes, $n=20$ and 200. The choices of the distributions used and the range choices for each distribution were chosen to use the idea of [13].

\section{Results and Discussions from the Simulation Study}

The Simulated results for the Hampel, Bisquare (Biweight), Alarm and Anekwe's redescending M-estimators are discussed as follows:

Discussion of stimulated results for data with outliers in both $x$ and $y$ directions.

At $10 \%$ outliers for both $x$ and $y$ axes in a simple regression, the result from Table 1 
indicates that the Anekwe's estimator takes the lead as the most efficient and robust method but followed closely by the Alarm estimator. The Hampel and Bisquare estimators performed badly in this category.

Since M-estimators cannot perform very well when outliers are in the $x$-direction, the results of Hampeland Bisquare estimators got worse by the increase of outliers at both axes, that is, $15 \%$ outliers for both $x$ and $y$ axes in a simple regression as shown in Table 2. The Anekwe estimator is still the best with respect to efficiency and robustness but followed closely by the Alarm estimator.

At $20 \%$ outliers in both axes in a simple regression as shown in Table 3, the Anekwe and Alarm estimators are more efficient and robust compared to other estimators. All the estimators do not perform very well in this category with Bisquare estimator on the lead.

Table 4 presents the result for $10 \%$ outliers in both axes in a multiple regression model. The Anekwe estimator is more efficient and robust compared to other estimators. Alarm and Hampel estimator follow closely as the second and third best estimators respectively. The Bisquare estimator has high parameters estimates for $\beta_{1}$.

Furthermore, the Anekwe estimator takes the lead as the most efficient and robust estimator as shown in Table 5 for $15 \%$ outliers for both $x$ and $y$ axes in a multiple regression. Alarm estimator came second while Hampel's estimator was the third most efficient and robust estimator. The Bisquare estimator is also the least efficient and robust estimator in this category.

Lastly, Table 6 presents the result for $20 \%$ outliers in both axes in a multiple regression model. The Anekwe estimator outperformed other estimators as the most efficient and robust estimator. The second most efficient and robust estimator is the Alarm estimator which performs better than Hampel and Bisquare estimators. 
Table 1: Simulated MSE and BIAS on simple regression for $10 \%$ outliers in $x$ and $y$-axes

\begin{tabular}{|c|l|l|l|l|l|l|}
\hline $\begin{array}{l}\text { Sample } \\
\text { Size }\end{array}$ & Beta & Criteria & Bisquare & Hampel & Alarm & Anekwe \\
\hline $\mathbf{2 0}$ & $\boldsymbol{\beta}_{\mathbf{0}}$ & BIAS & 0.0084 & 0.0913 & 0.0297 & 0.0130 \\
\hline & $\boldsymbol{\beta}_{\mathbf{0}}$ & MSE & 0.1651 & 0.1832 & 0.0903 & 0.0870 \\
\hline $\mathbf{2 0}$ & $\boldsymbol{\beta}_{\mathbf{1}}$ & BIAS & 1.9782 & 1.9831 & 0.3536 & 0.3026 \\
\hline & $\boldsymbol{\beta}_{\mathbf{1}}$ & MSE & 3.9194 & 3.9387 & 0.9525 & 0.8435 \\
\hline $\mathbf{2 0 0}$ & $\boldsymbol{\beta}_{\mathbf{0}}$ & BIAS & 0.0138 & 0.0733 & 0.0204 & 0.0092 \\
\hline & $\boldsymbol{\beta}_{\mathbf{0}}$ & MSE & 0.0161 & 0.0224 & 0.0079 & 0.0072 \\
\hline $\mathbf{2 0 0}$ & $\boldsymbol{\beta}_{\mathbf{1}}$ & BIAS & 1.9747 & 1.9812 & 0.0072 & 0.0064 \\
\hline & $\boldsymbol{\beta}_{\mathbf{1}}$ & MSE & 3.9000 & 3.9256 & 0.0252 & 0.0242 \\
\hline
\end{tabular}

Table 2: Simulated MSE and BIAS on simple regression for $15 \%$ outliers in $x$ and $y$-axes

\begin{tabular}{|l|l|l|l|l|l|l|}
\hline $\begin{array}{l}\text { Sample } \\
\text { Size }\end{array}$ & Beta & Criteria & Bisquare & Hampel & Alarm & Anekwe \\
\hline $\mathbf{2 0}$ & $\boldsymbol{\beta}_{\mathbf{0}}$ & BIAS & 0.0344 & 0.1965 & 0.0539 & 0.0203 \\
\hline & $\boldsymbol{\beta}_{\mathbf{0}}$ & MSE & 0.1939 & 0.2917 & 0.1469 & 0.1302 \\
\hline $\mathbf{2 0}$ & $\boldsymbol{\beta}_{\mathbf{1}}$ & BIAS & 1.9850 & 1.9965 & 0.5903 & 0.5300 \\
\hline & $\boldsymbol{\beta}_{\mathbf{1}}$ & MSE & 3.9452 & 3.9912 & 1.5426 & 1.3790 \\
\hline $\mathbf{2 0 0}$ & $\boldsymbol{\beta}_{\mathbf{0}}$ & BIAS & 0.0310 & 0.1949 & 0.0634 & 0.0290 \\
\hline & $\boldsymbol{\beta}_{\mathbf{0}}$ & MSE & 0.0216 & 0.0636 & 0.0138 & 0.0100 \\
\hline $\mathbf{2 0 0}$ & $\boldsymbol{\beta}_{\mathbf{1}}$ & BIAS & 1.9875 & 1.9983 & 0.0163 & 0.0270 \\
\hline & $\boldsymbol{\beta}_{\mathbf{1}}$ & MSE & 3.9504 & 3.9936 & 0.0649 & 0.0816 \\
\hline
\end{tabular}


Table 3: Simulated MSE and BIAS on simple regression for $20 \%$ outliers in $x$ and $y$-axes

\begin{tabular}{|c|l|l|l|l|l|l|}
\hline $\begin{array}{l}\text { Sample } \\
\text { Size }\end{array}$ & Beta & Criteria & Bisquare & Hampel & Alarm & Anekwe \\
\hline $\mathbf{2 0}$ & $\boldsymbol{\beta}_{\mathbf{0}}$ & BIAS & 0.0809 & 0.5218 & 0.1471 & 0.0571 \\
\hline & $\boldsymbol{\beta}_{\mathbf{0}}$ & MSE & 0.2606 & 0.9700 & 0.2870 & 0.2245 \\
\hline $\mathbf{2 0}$ & $\boldsymbol{\beta}_{\mathbf{1}}$ & BIAS & 1.9939 & 2.0231 & 1.0304 & 0.9843 \\
\hline & $\boldsymbol{\beta}_{\mathbf{1}}$ & MSE & 3.9797 & 4.0984 & 2.5685 & 2.3292 \\
\hline $\mathbf{2 0 0}$ & $\boldsymbol{\beta}_{\mathbf{0}}$ & BIAS & 0.0916 & 0.4125 & 0.1772 & 0.0743 \\
\hline & $\boldsymbol{\beta}_{\mathbf{0}}$ & MSE & 0.0354 & 0.2180 & 0.0547 & 0.0247 \\
\hline $\mathbf{2 0 0}$ & $\boldsymbol{\beta}_{\mathbf{1}}$ & BIAS & 1.9970 & 2.0180 & 0.3748 & 0.6731 \\
\hline & $\boldsymbol{\beta}_{\mathbf{1}}$ & MSE & 3.9885 & 4.0727 & 0.8216 & 1.4156 \\
\hline
\end{tabular}

Table 4: Simulated MSE and BIAS on multiple regression for $10 \%$ outliers in $x$ and $y$ axes

\begin{tabular}{|l|l|l|l|l|l|l|}
\hline $\begin{array}{l}\text { Sample } \\
\text { Size }\end{array}$ & Beta & Criteria & Bisquare & Hampel & Alarm & Anekwe \\
\hline $\mathbf{2 0}$ & $\boldsymbol{\beta}_{\mathbf{0}}$ & BIAS & 0.0007 & 0.0384 & 0.0131 & 0.0051 \\
\hline & $\boldsymbol{\beta}_{\mathbf{0}}$ & MSE & 0.1750 & 0.1096 & 0.1054 & 0.1072 \\
\hline $\mathbf{2 0}$ & $\boldsymbol{\beta}_{\mathbf{1}}$ & BIAS & 1.7762 & 0.3924 & 0.3628 & 0.3328 \\
\hline & $\boldsymbol{\beta}_{\mathbf{1}}$ & MSE & 3.5352 & 1.0484 & 0.9481 & 0.9278 \\
\hline $\mathbf{2 0}$ & $\boldsymbol{\beta}_{\mathbf{2}}$ & BIAS & 0.0162 & 0.0138 & 0.0021 & 0.0044 \\
\hline & $\boldsymbol{\beta}_{\mathbf{2}}$ & MSE & 0.1574 & 0.1004 & 0.0893 & 0.0100 \\
\hline $\mathbf{2 0 0}$ & $\boldsymbol{\beta}_{\mathbf{0}}$ & BIAS & 0.0154 & 0.0286 & 0.0107 & 0.0049 \\
\hline & $\boldsymbol{\beta}_{\mathbf{0}}$ & MSE & 0.0146 & 0.0109 & 0.0069 & 0.0064 \\
\hline $\mathbf{2 0 0}$ & $\boldsymbol{\beta}_{\mathbf{1}}$ & BIAS & 1.7170 & 0.5577 & 0.0656 & 0.0343 \\
\hline & $\boldsymbol{\beta}_{\mathbf{1}}$ & MSE & 3.0553 & 0.9027 & 0.0718 & 0.0473 \\
\hline $\mathbf{2 0 0}$ & $\boldsymbol{\beta}_{\mathbf{2}}$ & BIAS & 0.0246 & 0.0274 & 0.0036 & 0.0043 \\
\hline & $\boldsymbol{\beta}_{\mathbf{2}}$ & MSE & 0.0131 & 0.0120 & 0.0052 & 0.0049 \\
\hline
\end{tabular}


Table 5: Simulated MSE and BIAS on multiple regression for $15 \%$ outliers in $x$ and $y$ axes

\begin{tabular}{|l|l|l|l|l|l|l|}
\hline $\begin{array}{l}\text { Sample } \\
\text { size }\end{array}$ & Beta & Criteria & Bisquare & Hampel & Alarm & Anekwe \\
\hline $\mathbf{2 0}$ & $\boldsymbol{\beta}_{\mathbf{0}}$ & BIAS & 0.0680 & 0.1109 & 0.0407 & 0.0182 \\
\hline & $\boldsymbol{\beta}_{\mathbf{0}}$ & MSE & 0.5347 & 0.2455 & 0.1569 & 0.1462 \\
\hline $\mathbf{2 0}$ & $\boldsymbol{\beta}_{\mathbf{1}}$ & BIAS & 1.9679 & 0.5967 & 0.4631 & 0.4363 \\
\hline & $\boldsymbol{\beta}_{\mathbf{1}}$ & MSE & 4.0595 & 1.6517 & 1.2286 & 1.1852 \\
\hline $\mathbf{2 0}$ & $\boldsymbol{\beta}_{\mathbf{2}}$ & BIAS & 0.1498 & 0.1103 & 0.0369 & 0.0132 \\
\hline & $\boldsymbol{\beta}_{\mathbf{2}}$ & MSE & 0.4868 & 0.3000 & 0.1460 & 0.1378 \\
\hline $\mathbf{2 0 0}$ & $\boldsymbol{\beta}_{\mathbf{0}}$ & BIAS & 0.0122 & 0.1560 & 0.0297 & 0.0117 \\
\hline & $\boldsymbol{\beta}_{\mathbf{0}}$ & MSE & 0.0208 & 0.0553 & 0.0116 & 0.0091 \\
\hline $\mathbf{2 0 0}$ & $\boldsymbol{\beta}_{\mathbf{1}}$ & BIAS & 1.8639 & 1.3730 & 0.2419 & 0.0741 \\
\hline & $\boldsymbol{\beta}_{\mathbf{1}}$ & MSE & 3.5381 & 2.7039 & 0.3560 & 0.0787 \\
\hline $\mathbf{2 0 0}$ & $\boldsymbol{\beta}_{\mathbf{2}}$ & BIAS & 0.0999 & 0.2670 & 0.0148 & 0.0020 \\
\hline & $\boldsymbol{\beta}_{\mathbf{2}}$ & MSE & 0.0294 & 0.1325 & 0.0104 & 0.0063 \\
\hline
\end{tabular}

Table 6: Simulated MSE and BIAS on multiple regression for $20 \%$ outliers in $x$ and $y$ axes

\begin{tabular}{|l|l|l|l|l|l|l|}
\hline $\begin{array}{l}\text { Sample } \\
\text { size }\end{array}$ & Beta & Criteria & Bisquare & Hampel & Alarm & Anekwe \\
\hline $\mathbf{2 0}$ & $\boldsymbol{\beta}_{\mathbf{0}}$ & BIAS & 0.3308 & 0.6816 & 0.1465 & 0.0603 \\
\hline & $\boldsymbol{\beta}_{\mathbf{0}}$ & MSE & 1.0812 & 1.9598 & 0.4146 & 0.2479 \\
\hline $\mathbf{2 0}$ & $\boldsymbol{\beta}_{\mathbf{1}}$ & BIAS & 1.9990 & 1.0853 & 0.8032 & 0.6188 \\
\hline & $\boldsymbol{\beta}_{\mathbf{1}}$ & MSE & 4.1447 & 3.3236 & 1.9788 & 1.6481 \\
\hline $\mathbf{2 0}$ & $\boldsymbol{\beta}_{\mathbf{2}}$ & BIAS & 0.5267 & 0.5560 & 0.1276 & 0.0440 \\
\hline
\end{tabular}




\begin{tabular}{|l|l|l|l|l|l|l|}
\hline & $\boldsymbol{\beta}_{\mathbf{2}}$ & MSE & 1.5443 & 1.6220 & 0.4391 & 0.2376 \\
\hline $\mathbf{2 0 0}$ & $\boldsymbol{\beta}_{\mathbf{0}}$ & BIAS & 0.1171 & 0.8758 & 0.1856 & 0.0510 \\
\hline & $\boldsymbol{\beta}_{\mathbf{0}}$ & MSE & 0.0476 & 1.0360 & 0.0847 & 0.0192 \\
\hline $\mathbf{2 0 0}$ & $\boldsymbol{\beta}_{\mathbf{1}}$ & BIAS & 1.9319 & 1.9476 & 0.8974 & 0.2640 \\
\hline & $\boldsymbol{\beta}_{\mathbf{1}}$ & MSE & 3.7705 & 4.0105 & 1.8030 & 0.3753 \\
\hline $\mathbf{2 0 0}$ & $\boldsymbol{\beta}_{\mathbf{2}}$ & BIAS & 0.2784 & 1.0724 & 0.2496 & 0.0205 \\
\hline & $\boldsymbol{\beta}_{\mathbf{2}}$ & MSE & 0.1216 & 1.4194 & 0.1884 & 0.0161 \\
\hline
\end{tabular}

\section{Real-life Data}

For comparison, we applied the redescending M-estimators to real-life data and the dataset had been extensively used by other researchers in the area of robust regression.

\section{The Hawkins, Bradu, and Kass data}

[8] generated artificial data for testing the performance of robust estimators. The data contains 75 observations in four dimensions (one response and three explanatory variables). The first 10 observations are bad leverage points, and the next four points are good leverage points (i.e., their $\boldsymbol{x}_{\boldsymbol{i}}$ are outlying, but the corresponding $\boldsymbol{y}_{\boldsymbol{i}}$ fit the model quite well).

Table 7: Estimates of the model parameters for Hawkins, Bradu and Kass data

\begin{tabular}{|c|l|l|l|l|}
\hline Parameter & Hampel & Biweight & Alarm & Proposed \\
\hline$\beta_{\mathbf{0}}$ & -0.181 & -0.946 & -0.181 & -0.181 \\
\hline$\beta_{\mathbf{1}}$ & 0.081 & 0.145 & 0.082 & 0.081 \\
\hline$\beta_{\mathbf{2}}$ & 0.040 & 0.197 & 0.040 & 0.040 \\
\hline $\boldsymbol{\beta}_{\mathbf{3}}$ & -0.052 & 0.180 & -0.052 & -0.052 \\
\hline $\begin{array}{l}\text { Data points used } \\
\text { Residual }\end{array}$ & 65 & 71 & 65 & 65 \\
\hline
\end{tabular}


The summary of the results for estimates of the model parameters for Hawkins, Bradu and Kass data for the estimators are presented in Table 7. Alarm, Hampel and Anekwe's method detected 10 outliers in the robust fit while Biweight estimator detected 4 outliers in the analysis.

\section{Conclusion}

Simulation studies were done to ascertain the effectiveness of the redescending Mestimators when outliers are in both $x$ and $y$ directions. Mean square error (MSE) and BIAS were used for comparison under two different sample sizes.

From the stimulated results, it was obvious that when outliers are both in the leverage points and the response, the Anekwe estimator is the most efficient and robust estimator compared to the Hampel, Alarm and Bisquare estimators.

\section{References}

[1] A. A. Alamgir, S. A. Khan, D. M. Khan and U. Khalil, A new efficient redescending Mestimator: Alamgir redescending M-estimator, Research Journal of Recent Sciences 2(8) (2013), 79-91.

[2] D. F. Andrews, P. J. Bickel, F. R. Hampel, P. J. Huber, W. H. Rogers and J. W. Tukey, Robust Estimates of Location: Survey and Advances, Princeton, NJ: Princeton University Press, 1972.

[3] S. Anekwe and S. Onyeagu, The redescending M-estimator for detection and deletion of outliers in regression analysis, Pakistan Journal of Statistics and Operations Research 17(4) (2021), 997-1014. https://doi.org/10.18187/pjsor.v17i4.3546

[4] A. E. Beaton and J. W. Tukey, The fitting of power series, meaning polynomials, illustrated on band-spectroscopic data, Tecnometrics 16(2) (1974), 147-185. https://doi.org/10.1080/00401706.1974.10489171

[5] N. R. Draper and H. Smith, Applied Regression Analysis, 3rd ed., New York: John Wiley and Sons, 1998. https://doi.org/10.1002/9781118625590

[6] F. R. Hampel, The influence curve and its role in robust estimation, Journal of the American Statistical Association 69(346) (1974), 383-393.

https://doi.org/10.1080/01621459.1974.10482962

[7] F. R. Hampel, E. M. Ronchetti, P. J. Rousseeuw and W. A. Stahel, Robust Statistics the Approach Based on Influence Functions, New York: John Wiley, 1986. 
[8] D. M. Hawkins, D. Bradu and G. V. Kass, Location of several outliers in multiple regression data using elemental sets, Technometrics 26 (1984), 197-208. https://doi.org/10.1080/00401706.1984.10487956

[9] P. J. Huber, Robust estimation of location parameter, The Annals of Mathematical Statistics 35 (1964), 73-101. https://doi.org/10.1214/aoms/1177703732

[10] T. D. Nguyena and R. Welch, Outlier detection and least trimmed squares approximation using semi-definite programming, Comput. Stat. Data 54 (2010), 3212-3226. https://doi.org/10.1016/j.csda.2009.09.037

[11] P. J. Rousseeuw, Least Median of Squares Regression, Research Report No. 178, Centre for Statistics and Operations Research, VUB Brussels, 1982.

[12] P. J. Rousseeuw, Multivariate Estimation with High Breakdown Point, Research Report No. 192, Centre for Statistics and Operations Research, VUB Brussels, 1983.

[13] P. J. Rousseeuw and A. M. Leroy, Robust Regression and Outlier Detection, New York: Wiley-Interscience, 1987. https://doi.org/10.1002/0471725382

[14] J. W. Tukey, Exploratory Data Analysis, Reading, MA: Addison-Wesley, 1977.

This is an open access article distributed under the terms of the Creative Commons Attribution License (http://creativecommons.org/licenses/by/4.0/), which permits unrestricted, use, distribution and reproduction in any medium, or format for any purpose, even commercially provided the work is properly cited. 